\title{
EVALUACIÓN AGRONÓMICA Y ECONÓMICA DE ARVEJA ARBUSTIVA (Pisum sativum L.) EN DIFERENTES ÉPOCAS DE SIEMBRA Y SISTEMAS DE TUTORADO
}

\section{AGRONOMIC AND ECONOMIC EVALUATION OF PEA (Pisum sativum L.) TYPE ARBUSTIVE IN DIFFERENT SEASONS OF SOWING AND TRAINING SYSTEMS}

\author{
Óscar Eduardo Checa Coral ${ }^{1}$, Jhuliana Estefany Bastidas Acosta ${ }^{2}$, Olga Cristina Narváez Taimal ${ }^{3}$
}

\begin{abstract}
${ }^{1}$ I.A. Ph.D. Grupo de Investigación en Cultivos Andinos GRICAND, Facultad de Ciencias Agrícolas. Universidad de Nariño -sede Vipri-, Cra. 36 No. 5-121, bloque 5, oficina 408, San Juan de Pasto - Nariño, Colombia, e-mail: cicagrarias@hotmail. com; ${ }^{2}$ I.A. Grupo de Investigación en Cultivos Andinos GRICAND, Facultad de Ciencias Agrícolas. Universidad de Nariño sede Vipri-, Cra. 36 No. 5-121, bloque 5, oficina 408, San Juan de Pasto - Nariño, Colombia, e-mail: jhuly0609@gmail.com; ${ }^{3}$ I.A. Grupo de investigación en Cultivos Andinos GRICAND, Facultad de Ciencias Agrícolas. Universidad de Nariño -sede Vipri-, Cra. 36 No. 5-121, bloque 5, oficina 408, San Juan de Pasto - Nariño, Colombia, e-mail: crisnata.91@hotmail.com
\end{abstract}

Rev. U.D.C.A Act. \& Div. Cient. 20(2): 279- 288, Julio-Diciembre, 2017

RESUMEN

En Nariño, la zona cerealista, no cuenta con alternativas de rotación, que permita al agricultor mejorar sus ingresos, romper ciclos de patógenos y diversificar sus sistemas productivos; una posible opción podría ser la arveja arbustiva. En la granja Lope del Sena, seccional Nariño, se evaluó el efecto de época de siembra y sistemas de tutorado, sobre cinco genotipos de arveja arbustiva. Las épocas de siembra correspondieron a marzo (época I), abril (época II) y mayo (época III). En cada periodo, se utilizó un diseño de bloques completos al azar, con arreglo en parcelas divididas. La parcela principal correspondió a los sistemas de tutorado vertical (TV), horizontal (TH) y al testigo sin tutor (ST) y las subparcelas a cinco genotipos de arveja arbustiva. Las variables evaluadas fueron: días a floración, días a cosecha en verde, peso de vainas, número de vainas por planta, rendimiento y porcentaje de vainas sanas. Se determinó la viabilidad económica de los tratamientos utilizando el análisis de presupuesto parcial. Los resultados indicaron mayores rendimientos en las épocas II y III. Los sistemas TV y TH superaron al sistema ST en porcentaje de vainas sanas (\%VS). Las líneas UN6651 y UN5174 sobresalieron en peso de vaina y rendimiento. UN6651 obtuvo la mejor respuesta para \%VS. La mayor viabilidad económica en las épocas I y III fue para el sistema sin tutor y en la época II, para los sistemas de tutorado vertical y tutorado horizontal.

Palabras clave: Genotipo, ambiente, rendimiento, sanidad, rentabilidad.

\section{SUMMARY}

In the department of Nariño, the area cultivated in cereals has no rotation alternatives that allow farmers to improve their incomes, in addition to breaking pathogen cycles and diversifying their production systems. A possible option could be shrub pea. In SENA's Lope Farm sectional Nariño, the effect of sowing season and training systems on five genotypes of shrub pea was evaluated. The sowing season corresponded to march (season I), april (season II) and may (season III). A randomized complete block design was used in each period in divided plots. The main plot corresponded to vertical training system (TV), horizontal training system $(\mathrm{TH})$ and any training system (ST), and the subplots to five genotypes of shrub pea. The variables evaluated were: days at flowering, days at harvest in green, pod weight, number of pods per plant, yield and percentage of healthy pods. The viability of the treatments was determined using the partial budget analysis. The results indicated higher yields in seasons II and III. The TV and TH systems exceeded the ST in percentage of healthy pods (\%VS). Lines UN6651 and UN5174 stood out in pod green weight and yield. UN6651 got the best response for $\%$ VS. The greatest economic viability at seasons I and III was for the any training system and at season II for the systems vertical training and horizontal training.

Key words: Genotype, environment, yield, plant health, profitability. 


\section{INTRODUCCIÓN}

Nariño es el principal productor de arveja del país, con un promedio de 48.264t anuales, que representan el 55,2\% de la producción nacional (DANE, 2016) y se estima que de este cultivo dependen más de 26.000 productores (Pantoja et al. 2014). Las variedades de mayor uso en Nariño son: Obonuco Andina, San Isidro, Sureña y Sindamanoy, las cuales, requieren sistemas de tutorado, que representan cerca del 52\% de los costos de producción (Checa \& Rodríguez, 2015).

La zona cerealista de Nariño, ubicada entre los 2.000 y $2.800 \mathrm{msnm}$, no cuenta con alternativas de rotación, que le permita mejorar sus ingresos y una posible opción es la producción de arvejas arbustivas, que presenta, como ventajas: menores costos de producción, al no requerir tutorados (Casanova et al. 2012); genotipos precoces, con periodos vegetativos mínimos de hasta 90 días y uniformidad en la maduración del producto, que facilita los procesos de recolección y de cosecha en verde. Estas características permiten que las arvejas arbustivas sean incluidas en objetivos de evaluación y de adaptación para ser entregadas al agricultor, siendo una alternativa para las zonas trigueras o de reconversión, donde el productor no cuenta con suficientes recursos económicos.

A la fecha, no se han obtenido variedades mejoradas de arveja arbustiva para el consumo en fresco, en grano seco o para agroindustria en Colombia, siendo un campo de investigación que puede fortalecer los sistemas productivos de los agricultores de la zona fría, del departamento de Nariño. En evaluaciones de cultivares de arveja arbustiva, se estableció que la precocidad es una de las características varietales más sensibles a las condiciones climáticas de un sitio. De igual manera, la temperatura y la precipitación son factores determinantes en la producción y el desarrollo de la arveja, considerándose de gran efecto sobre el rendimiento y sus componentes (González \& Ligarreto, 2006). La temperatura óptima para el cultivo de arveja, se encuentra entre 13 y $18^{\circ} \mathrm{C}$, mientras que su requerimiento hídrico oscila entre 250 y 380mm de agua bien distribuidos durante el ciclo de cultivo, con mayor demanda durante la etapa de crecimiento y de floración (FENALCE, 2006).

En la búsqueda de arvejas arbustivas, como alternativa productiva, es necesario identificar genotipos de alto rendimiento, determinar la época de siembra más adecuada para su cultivo y establecer la viabilidad del uso de algún tipo de tutorado sencillo, que evite el deterioro de las vainas por enfermedades, como el complejo ascoquita, producido por Ascochyta spp. y oidio, producido por Erysiphe polygoni. Lo anterior motivó la realización de la presente investigación.

\section{MATERIALES Y MÉTODOS}

Localización. La investigación, se llevó a cabo en las instalaciones de la Granja Lope del Sena, ubicada en el municipio de San Juan de Pasto, departamento de Nariño, a una altitud de $2.612 \mathrm{msnm}, 01^{\circ} 12^{\prime} 28^{\prime \prime} \mathrm{N}, 77^{\circ} 15^{\prime} 06^{\prime \prime} \mathrm{W}$, precipitación promedio anual de $814 \mathrm{~mm}$, temperatura promedio anual de $13^{\circ} \mathrm{C}$ y humedad relativa de $73 \%$ (IDEAM, 2016).

Diseño experimental. La evaluación incluyó tres épocas de siembra, dos sistemas de tutorado junto al testigo sin tutor y cinco genotipos de arveja arbustiva. Las épocas de siembra correspondieron a la primera semana de marzo, abril y mayo de 2016. En cada época de siembra, se estableció un diseño de bloques completos al azar, con arreglo en parcelas divididas, asignándose -en la parcela principal- los sistemas de tutorado y, en las sub-parcelas, los genotipos de arveja.

En los sistemas de tutorado, se incluyeron los tratamientos de tutorado vertical (TV), tutorado horizontal (TH) o encanastillado y sin tutorado (ST), como testigo. Para establecer los sistemas de tutorado horizontal y vertical, en las unidades experimentales se colocaron estacas con altura, a partir del nivel del suelo, de $1,2 \mathrm{~m}$ y enterradas a una profundidad de $0,2 \mathrm{~m}$. Para el tutorado vertical, en la parte superior de las estacas, se templó fibra plástica gruesa (diámetro 2,5mm) en forma horizontal y las plantas se amarraron con fibra de polipropileno delgada (diámetro $0,5 \mathrm{~mm}$ ), desde la base de la planta hasta el hilo horizontal, que se encontraba templado en la parte superior. Para el tutorado horizontal, una vez ubicadas las estacas en el inicio y final de cada surco, se templaron hilos de polipropileno delgado (diámetro 1,5mm), de forma horizontal, a lado y lado de las plantas, realizándose hasta cuatro hiladas en función al crecimiento de las plantas. Los genotipos de arveja arbustiva evaluados fueron: ILS3566, ILS3572, UN5173, UN5174 y UN665, pertenecientes a la colección de trabajo del programa de mejoramiento genético del Grupo de Investigación en Cultivos Andinos GRICAND, de la Facultad de Ciencias Agrícolas, de la Universidad de Nariño, sede Pasto.

La unidad experimental estuvo constituida por cuatro surcos de $3 \mathrm{~m}$ de largo, a una distancia de $0,5 \mathrm{~m}$ entre surco y $0,10 \mathrm{~m}$ entre sitio, colocando una semilla por sitio y dejando calle de $1 \mathrm{~m}$, cada cuatro surcos, para facilitar el manejo agronómico, obteniendo una densidad de 160.000 plantas. ha $^{-1}$. La parcela útil correspondió a los dos surcos centrales de la unidad experimental, descartando los surcos borde y las plantas extremas de cada uno. La respuesta de los sistemas de tutorado y los genotipos en cada época, se contrastaron con variables climáticas de precipitación y de temperatura registradas por la estación meteorológica del Sena, en San Juan de Pasto, en donde se desarrolló la presente investigación. 
Manejo agronómico. Las labores de cultivo, se realizaron siguiendo las recomendaciones de Buitrago et al. (2006). La fertilización, se efectuó a los 15 días después de la siembra, aplicando $7 \mathrm{~g}$ por planta de 10-30-10, más un fertilizante compuesto de micronutrientes y de nutrientes secundarios, en proporción 5:1. Para el control de malezas, se aplicó un herbicida de ingrediente activo: metribuzina, en dosis de 400 $\mathrm{mm} \cdot \mathrm{ha}^{-1}$. Por último, se realizó una deshierba manual.

Variables evaluadas. Días a floración (DF): desde la fecha de siembra hasta cuando el $50 \%$ de las plantas presentó la primera flor abierta; días a cosecha en verde (DC): desde la fecha de siembra hasta cuando el $75 \%$ de las plantas presentó las vainas con madurez de cosecha en fresco; altura de la planta (AP): tomada en cinco plantas del área útil y obteniendo su promedio en $\mathrm{cm}$; número de vainas por planta (NVP): resultante del conteo de vainas realizado en todas las plantas de la parcela útil y sacando el promedio; peso de vaina verde (PV): tomado en 10 vainas de cada unidad experimental y sacando su promedio en gramos; rendimiento en vaina verde (RTO): tomando la producción obtenida en la parcela útil y llevándola a t.ha ${ }^{-1}$; porcentaje de vainas sanas (\%VS): se obtuvo después de separar las vainas sanas de las vainas afectadas por ascoquita (Ascochyta pisi), antracnosis (Colletotrichum pisi) y oidio (Erysiphe polygoni), llevando luego los datos a porcentaje. Las labores de cosecha para la toma de datos, se realizaron en tres pases.

Análisis estadístico. Los datos obtenidos fueron sometidos a un análisis de varianza combinado, considerando efectos fijos para todos los factores estudiados (época, tutorado y genotipo). Se tuvo en cuenta la significancia de los factores antes mencionados y sus interacciones. Además, se realizaron pruebas de comparación de medias de Tukey $\mathrm{P}<0,05$, para las variables que mostraron diferencias estadísticas en el Anava.

Análisis económico. Para determinar los tratamientos de mayor viabilidad económica, se utilizó la metodología de presupuesto parcial (Hernández, 2002). Para tal fin, se calcularon los costos de producción de los sistemas de tutorado en cada época de siembra, de acuerdo a lo observado en la investigación. De igual manera, se llevó a cabo la determinación de ingreso total (IT), a partir del rendimiento ajustado al porcentaje de vainas sanas, asignando el valor comercial del mercado, según su nivel de sanidad. Se realizó el análisis de dominancia de los tratamientos, a partir de sus costos variables de producción y su ingreso neto parcial (IN= IT$\mathrm{CV}$, donde $\mathrm{IN}=$ ingreso neto; $\mathrm{IT}=$ ingreso total; $\mathrm{CV}=$ costos variables). Los tratamientos no dominados fueron seleccionados y analizados desde el punto de vista económico, teniendo en cuenta la tasa de retorno marginal, que resulta de dividir el incremento en ingreso neto sobre el incremento en el costo variable, utilizando la siguiente fórmula: $T R M=100$ x ( $\Delta \mathrm{IN} / \Delta C V)$ (Hernández, 2002).

\section{RESULTADOS Y DISCUSIÓN}

Análisis de varianza. Se encontraron diferencias significativas $(p<0.001$ y $p<0,05)$ para los efectos simples de época, en las variables DC, AP, NVP, PV y RTO; tutorado en la variable \%VS y línea en DC, AP, NVP, RTO y \%VS. También, se registraron diferencias significativas para la interacción doble de época por línea, en los caracteres DC, AP, NVP, PV, RTO y \%VS; no se presentó significancia para la interacción triple época por tutorado por línea (Tabla 1). Para el análisis y la discusión de resultados, los efectos simples significativos, solo se tuvieron en cuenta en las variables que no mostraron significancia estadística en las distintas interacciones. La variable días a floración (DF) no se vio afectada por los factores época, tutorado, línea y sus interacciones, lo cual, sugiere que esta variable fue poco afectada por las variaciones bióticas y abióticas presentes en el periodo de evaluación.

Días a cosecha en verde (DC). En la interacción época por línea, se observó que, en la época I, el genotipo UN5173 mostró diferencias estadísticas con UN5174. En la época II, UN5173 superó a las demás líneas; ILS3566, por su parte, fue más tardía que ILS3572 y UN5174. En la época III, no se presentaron diferencias significativas (Tabla 2).

Casanova et al. (2012) afirman que el período vegetativo en las especies cultivadas está altamente influenciado por la constitución genética de las plantas, existiendo genotipos precoces y tardíos, característica importante para la selección de un material; en este caso, el material con mayor precocidad fue UN5174. En general, en la época I, los genotipos mostraron mayor precocidad que en las épocas II y III, lo cual, se asocia a la menor precipitación observada en el primer mes de cultivo de la época I y su mayor temperatura durante todo su ciclo respecto a las otras dos épocas de siembra (Figura 1). Lo anterior, se puede explicar si se tiene en cuenta que la disminución del ciclo de cultivo se puede dar por acumulación de grados día. De igual manera, en la época III, los genotipos evaluados fueron más precoces que en la época II, debido a la menor precipitación presentada en el último mes de cultivo de la época III.

Altura de planta (AP). La interacción época por línea mostró que en la época I, el genotipo UN5173 presentó diferencias significativas frente a UN6651. En la época II, UN5173 mostró mayor altura con respecto a los demás genotipos. En la época III, nuevamente UN5173 registró diferencias estadísticas frente a las demás líneas; UN5174 con $81,62 \mathrm{~cm}$, obtuvo un mayor promedio de altura que ILS3572 (Tabla 2). 
Tabla 1. Cuadrados medios del Anava para características y componentes de rendimiento en la evaluación agronómica, de cinco líneas de arveja arbustiva, bajo tres sistemas de tutorado, en tres épocas de siembra. San Juan de Pasto, 2016.

\begin{tabular}{|l|l|l|l|l|l|l|l|l|}
\hline Fuente de Variación & gl & DF & DC & AP & NVP & PV & RTO & \%VS \\
\hline Modelo & 79 & 195,56 & 187,46 & 1290,55 & 322,21 & 2,34 & 33,69 & 500,97 \\
\hline Épocas (E) & 2 & 1738,14 & $3062,96 * *$ & $24019,96 * *$ & $4437,01 * *$ & $15,3 * *$ & $408,81^{* *}$ & 132,83 \\
\hline Bloques & 2 & 604,05 & 100,84 & 230,22 & 471,89 & 0,64 & 94,6 & 55,97 \\
\hline Error A & 4 & 348,9 & 23,93 & 51,5 & 80,74 & 0,56 & 15,2 & 250,29 \\
\hline Tutorados (T) & 2 & 100,31 & 2,16 & 221,61 & 18,52 & 1,09 & 19,18 & $6668,76^{* *}$ \\
\hline Error B & 4 & 131,27 & 90,46 & 120,74 & 52,61 & 0,19 & 3,35 & 132,72 \\
\hline Líneas (L) & 4 & 263,31 & $515,04 * *$ & $1240,35^{* *}$ & $733,82^{* *}$ & 18,34 & $18,01^{*}$ & $2222,47^{* *}$ \\
\hline LxT & 8 & 134,03 & 62,53 & 45,69 & 25,94 & 0,97 & 10,15 & 234,16 \\
\hline Error C & 24 & 109,08 & 53,27 & 37,19 & 26,11 & 0,56 & 5,15 & 235,83 \\
\hline ExT & 4 & 100,1 & 57,51 & 95,67 & 58,59 & 0,52 & 8,44 & 56,07 \\
\hline ExL & 8 & 112,17 & $145,69 * *$ & $130,83 * *$ & $347,28 * *$ & $2,17 * *$ & $24,32 * *$ & $264,06 *$ \\
\hline ExTxL & 16 & 110 & 36,24 & 52,7 & 35,23 & 0,71 & 4,76 & 124,54 \\
\hline Número de plantas & 1 & 654,9 & 59,21 & 5,29 & 1133,73 & 0,1 & 55,86 & 3,49 \\
\hline Error D & 55 & 87,52 & 31,11 & 42,73 & 51,44 & 0,51 & 6,58 & 179,76 \\
\hline R & & 0,76 & 0,90 & 0,98 & 0,90 & 0,87 & 0,88 & 0,80 \\
\hline CV (\%) & & 15,14 & 5,43 & 10,91 & 26,41 & 11,99 & 26,35 & 19,01 \\
\hline Vxy & & $-0,51$ & $-0,15$ & $-0,05$ & $-0,67$ & 0,01 & 0,15 & 0,04 \\
\hline
\end{tabular}

$*=$ diferencias significativas $(\mathrm{p} \leq 0,05) ; * *=$ diferencias altamente significativas $(\mathrm{p} \leq 0,01)$.

DF: días a floración; DC: días a cosecha en vaina verde; AP: altura de planta (cm); NVP: número de vainas por planta; PV: peso de vaina verde (g); RTO: rendimiento (ton. ha ${ }^{-1}$ ); \%VS: porcentaje de vainas sanas; $\mathrm{R}^{2}$ : coeficiente de determinación; CV: coeficiente de variación; Vxy: covarianza.

En las tres épocas de siembra evaluadas, el genotipo UN5173 presentó mayor altura, mientras que UN6651 siempre estuvo entre los de porte más bajo; lo anterior está relacionado con la constitución genética de los materiales mencionados. Las líneas evaluadas tuvieron menor altura de planta en la época I, debido a la baja humedad a la que se vio enfrentado el cultivo en el primer mes después de la siembra, lo cual, pudo afectar la capacidad de absorción y de translocación de nutrientes en las plantas, reduciendo su desarrollo. Al respecto, Moreno (2009) y Bénézit et al. (2017) indican que las plantas con estrés hídrico presentan alteraciones en procesos fisiológicos y metabólicos, como reducción en las tasas de fotosíntesis, disminución de la síntesis de proteínas totales y de las tasas de crecimiento. Los resultados confirman las observaciones realizadas por Georgieva et al. (2015), quienes encontraron diferencias significativas para altura de planta en sus estudios de adaptación y de estabilidad en el cultivo de arveja, obteniendo una reacción altamente influenciada por el ambiente, para este carácter.
Número de vainas por planta (NVP). En la época I, las líneas UN5173, ILS3572 e ILS3566 fueron significativamente diferentes a UN5174 y UN6651, para número de vainas por planta. En la época II, los genotipos UN6651 y UN5173 mostraron diferencias significativas frente a ILS3566, ILS3572 y UN5174. En la época III, UN5173 fue superior a UN5174 (Tabla 2).

La mejor respuesta de las líneas UN5173, ILS3572 e ILS3566, bajo las condiciones presentadas en la época I (Tabla 2) (Figura 1) sugiere que, en condiciones de baja precipitación en el primer mes del cultivo, estas líneas tuvieron una mejor expresión genética para este carácter respecto a los otros genotipos evaluados. De igual manera, UN5173 y UN6651 obtuvieron promedios de NVP superiores en las épocas II y III, donde las condiciones ambientales fueron de alta precipitación, lo cual, permitió la mejor expresión de su potencial productivo. 
Tabla 2. Promedios para la interacción época por línea para las variables días a cosecha en verde (DC), altura de planta (AP) y número de vainas por planta (NVP).

\begin{tabular}{|c|c|c|c|c|c|c|c|c|c|}
\hline \multirow{2}{*}{ LÍNEA } & \multicolumn{3}{|c|}{ DC } & \multicolumn{3}{|c|}{ AP (cm) } & \multicolumn{3}{|c|}{ NVP } \\
\hline & Época I & Época II & Época III & Época I & Época II & Época III & Época I & Época II & Época III \\
\hline UN5173 & $96,09 \mathrm{~A}$ & $124,28 \mathrm{~A}$ & $107,88 \mathrm{~A}$ & $27,94 \mathrm{~A}$ & $88,99 \mathrm{~A}$ & $96,73 \mathrm{~A}$ & $20,57 \mathrm{~A}$ & $43,28 \mathrm{~A}$ & $37,5 \quad \mathrm{~A}$ \\
\hline ILS3566 & $92,02 \quad A B$ & $113,87 \mathrm{~B}$ & $107,34 \mathrm{~A}$ & $22,31 \quad A B$ & $72,19 \mathrm{~B}$ & $75,05 \mathrm{BC}$ & $15,77 \mathrm{~A}$ & $33,03 \mathrm{~B}$ & $35,96 \mathrm{AB}$ \\
\hline ILS3572 & $90,71 \mathrm{AB}$ & $106,16 \mathrm{C}$ & $106,47 \mathrm{~A}$ & $20,88 \mathrm{AB}$ & 74,19 B & $70,28 \mathrm{C}$ & $15,99 \mathrm{~A}$ & 29,82 B & $32,41 \mathrm{AB}$ \\
\hline UN6651 & $90,06 \mathrm{AB}$ & $108,31 \mathrm{BC}$ & $105,39 \mathrm{~A}$ & $18,37 \mathrm{~B}$ & $71,36 \mathrm{~B}$ & $74,51 \mathrm{BC}$ & $2,15 \quad \mathrm{~B}$ & $46,93 \mathrm{~A}$ & $35,98 \mathrm{AB}$ \\
\hline UN5174 & $87,57 \mathrm{~B}$ & $101,60 \mathrm{C}$ & $102,7 \quad \mathrm{~A}$ & $24,83 \mathrm{AB}$ & $79,54 \mathrm{~B}$ & 81,62 B & $5,27 \quad \mathrm{~B}$ & $25,32 \mathrm{~B}$ & $27,36 \mathrm{~B}$ \\
\hline $\begin{array}{l}\text { Medias } \\
\text { época }\end{array}$ & $91,29 \mathrm{C}$ & $110,84 \mathrm{~A}$ & 105,96 В & $22,86 \mathrm{~B}$ & $77,25 \mathrm{~A}$ & $79,64 \mathrm{~A}$ & 11,95 B & $35,67 \mathrm{~A}$ & $33,84 \mathrm{~A}$ \\
\hline
\end{tabular}

Medias con letras distintas en la misma columna indican diferencias significativas según la prueba de Tukey $(P \leq 0,05)$.

Diferencia honesta significativa de Tukey para días a cosecha $(D C)=7,45$; altura de planta $(A P)=8,74$ y número de vainas por planta $(\mathrm{NVP})=9,57$.

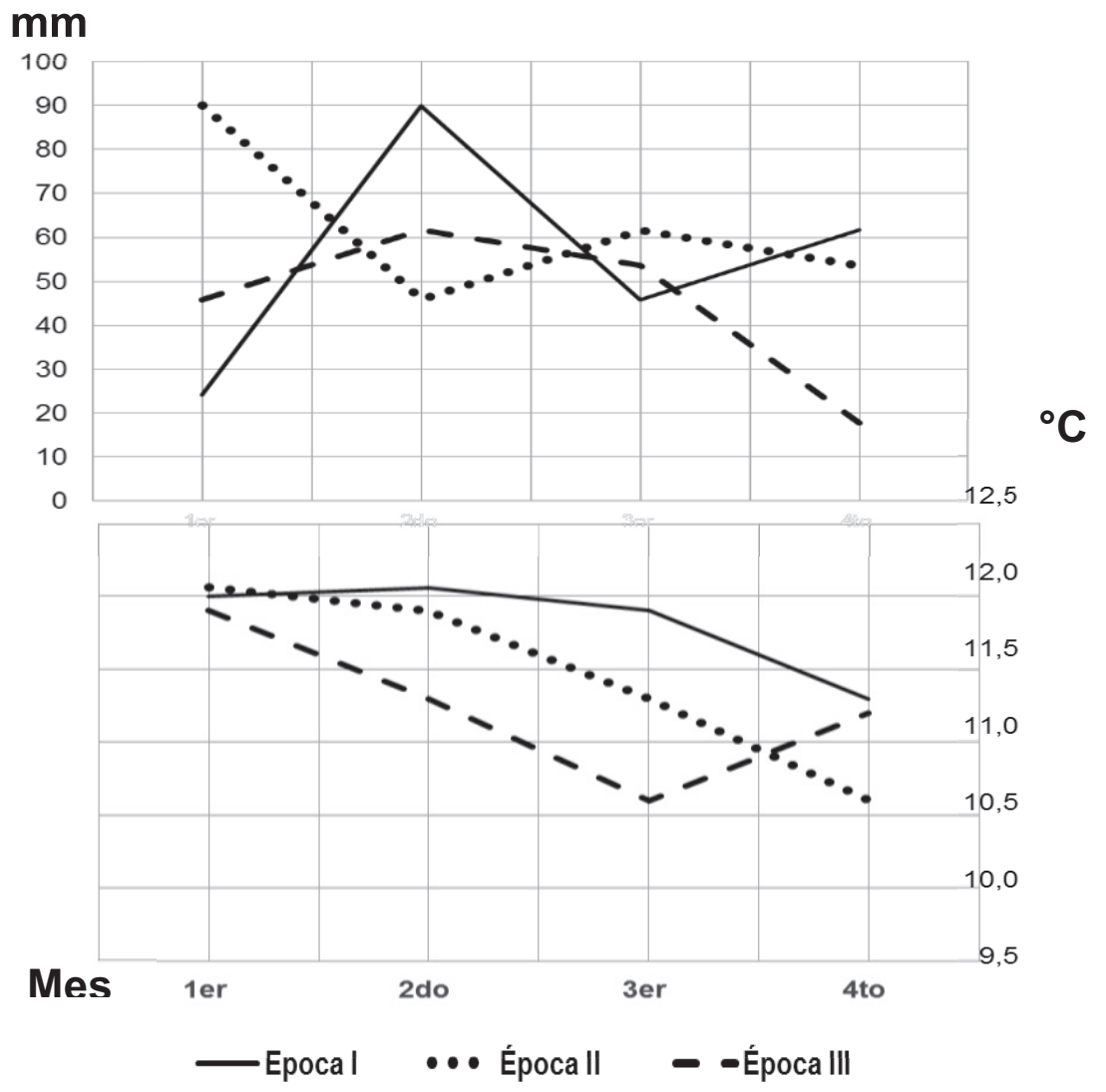

Figura 1. Precipitación y temperatura promedio para el ciclo de cultivo en la época de siembra I (marzo); época II (abril) y época III (mayo). 
Es importante resaltar que la línea UN5173 obtuvo el mayor promedio de altura y alta prolificidad en la producción de vainas por planta en las tres épocas, sugiriendo una asociación entre estas variables. Esto es posible, si se tiene en cuenta que, a mayor altura de planta, se puede aumentar la presencia de nudos reproductivos y, en consecuencia, el número de vainas por planta. Lo anterior confirma los resultados obtenidos por Kumar \& Roopa (2014) y Esmail et al. (2015), quienes encontraron que la altura de la planta esta positivamente correlacionada con el número de vainas por planta.

Peso de vaina verde (PV). La interacción época por línea mostró que, en la época I, la línea UN5174 presentó diferencias significativas frente a los demás genotipos; de igual manera, UN6651 superó a ILS3572 e ILS3566. En la época II sobresalieron UN5174 y UN6651, superando a ILS3572, UN5173 e ILS3566. En la época III, UN5174 obtuvo diferencias estadísticas frente a UN5173 e ILS3566; sobresalió también UN6651, que superó a ILS3566 (Tabla 3).
En las tres épocas, las líneas UN5174 y UN6651 se destacaron para peso de vaina con grano, característica que es de especial interés para los productores de esta leguminosa. Según Hallauer et al. (2010), el genotipo ideal debe tener el mayor rendimiento u otros rasgos de interés en un amplio rango de ambientes mostrando adaptabilidad general; sin embargo, también es posible seleccionar genotipos con adaptación específica, para determinadas localidades.

Por otra parte, aunque UN5174 mostró un alto promedio de PV, presentó también los menores promedios en NVP. Lo anterior es explicable, si se tiene en cuenta que en los componentes de rendimiento se presentan compensaciones $\mathrm{y}$, con frecuencia, el mejoramiento de una característica conduce a la reducción de otra. Se han encontrado compensaciones entre los componentes de rendimiento (Egli, 2017), por lo tanto, no es inesperado que la selección basada en componentes individuales falle al intentar incrementar el rendimiento total. En este mismo sentido, Pacheco et al. (2009)

Tabla 3. Promedios para la interacción época por línea para las variables peso de vaina verde (PV), rendimiento (RTO) y porcentaje de vainas sanas (\%VS).

\begin{tabular}{|c|c|c|c|c|c|c|c|c|c|}
\hline \multirow{2}{*}{ LIINEA } & \multicolumn{3}{|c|}{ PV (g) } & \multicolumn{3}{|c|}{ RTO (ton.ha ${ }^{-1}$ ) } & \multicolumn{3}{|c|}{$\% \mathrm{VS}$} \\
\hline & Época I & Época II & Época III & Época I & Época II & Época III & Época I & Época II & Época III \\
\hline UN5174 & $7,95 \mathrm{~A}$ & $7,83 \mathrm{~A}$ & 6,19 A & $5,90 \mathrm{AB}$ & $14,70 \mathrm{AB}$ & $10,56 \mathrm{AB}$ & $78,21 \mathrm{~A}$ & 66,79 В & $62,08 \quad \mathrm{~B}$ \\
\hline UN6651 & $6,09 \mathrm{~B}$ & $7,80 \mathrm{~A}$ & $5,48 \mathrm{AB}$ & $5,64 \mathrm{AB}$ & $16,67 \mathrm{~A}$ & $13,91 \mathrm{~A}$ & $82,94 \mathrm{~A}$ & $93,96 \quad$ A & $89,05 \mathrm{~A}$ \\
\hline UN5173 & $5,61 \mathrm{BC}$ & $5,54 \mathrm{~B}$ & $4,71 \mathrm{BC}$ & $8,41 \mathrm{~A}$ & $12,59 \mathrm{BC}$ & $9,57 \quad \mathrm{AB}$ & $66,97 \mathrm{~A}$ & $57,90 \quad$ В & $55,58 \quad B$ \\
\hline ILS3572 & $5,28 \mathrm{C}$ & $6,15 \mathrm{~B}$ & $5,39 \mathrm{ABC}$ & $4,44 \mathrm{~B}$ & $9,96 \quad \mathrm{C}$ & $7,59 \quad \mathrm{~B}$ & 66,49 A & $63,89 \quad \mathrm{~B}$ & $66,06 \quad \mathrm{~B}$ \\
\hline ILS3566 & $5,05 \mathrm{C}$ & $5,30 \mathrm{~B}$ & $4,56 \mathrm{C}$ & $5,84 \mathrm{AB}$ & $10,87 \mathrm{BC}$ & $9,42 \quad$ B & $68,80 \mathrm{~A}$ & $72,91 \quad \mathrm{AB}$ & 66,16 B \\
\hline $\begin{array}{l}\text { Media } \\
\text { época }\end{array}$ & $6,00 \mathrm{~A}$ & $6,53 \mathrm{~A}$ & $5,27 \mathrm{~B}$ & $6,04 \mathrm{~B}$ & $12,96 \mathrm{~A}$ & $10,21 \mathrm{~A}$ & - & - & - \\
\hline
\end{tabular}

Medias con letras distintas en la misma columna indican diferencias significativas según la prueba de Tukey $(P \leq 0,05)$. Diferencia honesta significativa de Tukey para peso de vaina verde $(P V)=0,9$; rendimiento $(R T O)=3,43$ y porcentaje de vainas sanas $(\% \mathrm{VS})=22,43$.

afirman que, con seguridad, el incremento en una o más características deseables traerá consigo la pérdida en otras, por lo que no se debe esperar la consecución de plantas de porte bajo altamente rendidoras, con un gran número vainas y semillas grandes, sino un balance de estas características. En arveja se han reportado compensaciones entre el número de vainas por planta y el número de granos por vaina (Moot \& Mcneil, 1995) y entre número de semillas por vaina y peso de la semilla (Ligarreto et al. 2009).

Los mayores promedios presentados por los genotipos en las épocas I y II para PV están relacionados con la mayor disponibilidad de agua en el periodo de formación de vainas y llenado de grano, es decir, en el tercer y cuarto mes del cultivo (Figura 1), mientras que en la época III, las plantas estuvieron expuestas a condiciones secas, para el mismo periodo, que es modal en la zona.

Rendimiento (RTO). Para la interacción época por línea, en la época I, UN5173 mostró diferencias significativas respecto a ILS3572. En la época II, sobresale la línea UN6651, superando a UN5173, ILS3566 e ILS3572; de igual manera, UN5174 obtuvo mayor rendimiento que ILS3572. En la época III, UN6651 superó a ILS3566 e ILS3572, respectivamente (Tabla 3).

El mejor comportamiento de UN5173 en la época I con respecto a las épocas II y III, indica que es un genotipo que res- 
ponde bien a condiciones de estrés por sequía en las primeras etapas del cultivo (Figura 1); esta misma línea obtuvo el mayor promedio de NVP, lo cual, pudo contribuir a su mayor rendimiento. Rasaei et al. (2011) y Goa \& Ashamo (2014) hallaron una correlación positiva entre el rendimiento y número de vainas por planta. En las épocas II y III sobresalió la línea UN6651, mientras que en la época I obtuvo bajo rendimiento; el resultado sugiere, que UN5173 responde mejor a la primera condición ambiental (época I), mientras que UN6651 es la de mejor respuesta en las otras dos (épocas II y III), caracterizadas por mayor precipitación desde el inicio del cultivo hasta la época de formación de vainas.

Por otra parte, la línea UN5174, a pesar de presentar el menor número de vainas por planta, estuvo entre los mejores promedios de rendimiento en las tres épocas evaluadas, mostrándose promisoria por su consistencia, a través de los ambientes; en contraste, UN5173, que obtuvo un alto NVP en las tres épocas, solo logró alto rendimiento en la época I. Es posible que la energía requerida para lograr un mayor número de vainas por planta, en algunos genotipos, genere un desequilibrio, principalmente, si en ciertas condiciones ambientales se presenta una desadaptación, que provoque la disminución del rendimiento esperado.

Khan et al. (2013), en sus estudios de comportamiento morfológico de genotipos de arveja bajo condiciones lluviosas, encontraron rendimientos con un rango entre 3,74 a 10,43 t.ha ${ }^{-1}$; lo anterior se cumplió para el promedio general de las épocas I y III, con 6,04 y 10,21t.ha ${ }^{-1}$, respetivamente; sin embargo, en la época II, con 12,96t.ha ${ }^{-1}$ los genotipos, superaron este rango. Trabajos de investigación para arvejas arbustivas reportan resultados de rendimiento que van desde los más bajos, como los obtenidos por Mishra (2014), de 5,5 t.ha $^{-1}$, hasta los más altos, como los reportados por Celis \& Prett (1995), de 20t.ha ${ }^{-1}$.

Porcentaje de vainas sanas (\%VS). La interacción época por línea mostró que en la época I, los genotipos no presentaron diferencias estadísticas; en la época II, la línea UN6651 mostró mayor porcentaje de sanidad frente a UN5174, ILS3572 y UN5173 y en la época III, UN6651 continuó presentando el mejor porcentaje de vainas sanas, superando a las demás líneas (Tabla 3).

En las tres épocas de siembra evaluadas, se observó la presencia de diferentes fitopatógenos que afectaron la calidad de las vainas. En la época I, se encontraron periodos de baja humedad al inicio del ciclo del cultivo, pero altas precipitaciones al final del mismo, favoreciendo el ataque del complejo de enfermedades foliares causadas por los patógenos, como Ascochyta pisi y Colletotrichum pisi (Valencia et al. 2012). Para las épocas II y III, las constantes precipitaciones alternadas con días soleados en la etapa de llenado de grano (4to mes), favorecieron en mayor grado el ataque de Erysiphe polygoni, en los genotipos más susceptibles. Este patógeno, se ve influenciado por las variaciones de temperatura con presencia de días lluviosos y veranillos cortos (Checa \& Rodríguez, 2015). Según Mera et al. (2007), la elevada incidencia a enfermedades en ambientes altamente húmedos, conlleva a correr el riesgo de perder una alta proporción de la producción por deterioro de vainas. El mejor comportamiento de UN6651 en porcentaje de sanidad de vainas en comparación con los demás genotipos, permite considerarlo como material promisorio para futuras evaluaciones, por reacción a E. polygoni.

Por otra parte, en la fuente de variación tutorado, tanto el sistema vertical (TV) como el sistema horizontal $(\mathrm{TH})$, presentaron el mejor porcentaje de sanidad, superando al testigo sin tutor (ST) (Tabla 4). Los resultados demuestran que los sistemas de tutorado vertical (TV) y horizontal (TH), a diferencia del testigo sin tutor (ST), son una interesante alternativa para lograr vainas más sanas bajo condiciones de alta humedad, al evitar el contacto de las vainas con el suelo, siendo menos afectadas por los patógenos fungosos.

Análisis económico. Se realizó, con base en los resultados de rendimiento y de sanidad de vainas de los sistemas de tutorado y el sistema sin tutor en cada una de las tres épocas evaluadas. En el cálculo de los costos variables, se incluyeron los valores de fibra, postes y mano de obra, requeridos en los sistemas de tutorado evaluados, así como también los costos de capital, teniendo en cuenta la tasa de interés anual de 33,5\% que, tomada para los cuatro meses de manejó del cultivo, fue del $11,7 \%$.

Ingreso total e ingreso neto. Al no observar diferencias estadísticas para el factor tutorado en la variable rendimiento (Tabla 2), se tomó el mismo valor correspondiente al promedio en los tres sistemas y se ajustó por el porcentaje de vainas sanas (\%VS), obtenido para cada época (Tabla 5). El valor alcanzado se multiplicó por el precio en el mercado, equivalente a $\$ 2.308 / \mathrm{kg}$ de arveja en vaina verde con buena calidad.

Análisis de dominancia. Para las épocas I y III, el sistema vertical (TV) y horizontal $(\mathrm{TH})$ resultaron dominados, debido a que el testigo sin tutor (ST) obtuvo mayor ingreso neto, sin aumentar sus costos variables. Para la época II, todos los sistemas resultaron no dominados (Tabla 5).

Tasa de retorno marginal (TRM). Para las épocas I y III, en el sistema sin tutor, los ingresos netos de producción de arveja en verde fueron superiores, en comparación con el testigo sin tutor, haciendo de este el sistema más viable económicamente, en comparación con los sistemas de tutorado horizontal y vertical, por lo tanto, no fue necesario realizar 
el análisis de la tasa de retorno marginal. En la época II, el análisis económico del presupuesto parcial indicó que, una opción económica favorable para agricultores con recursos limitados, fue el manejo del tratamiento sin tutor, el cual, alcanzó un beneficio neto parcial, de $\$ 16.338 .459$, sin costos variables por tutorado; sin embargo, si el agricultor está en condiciones económicas para adoptar una nueva tecnología, incrementando los costos variables en \$4.154.346, puede acceder a la siembra de materiales arbustivos bajo el sistema de tutorado horizontal, aumentando sus ingresos netos en \$3.036.603 sobre los ingresos ya obtenidos en el sistema sin tutor, con una tasa de retorno marginal de $73 \%$ (Tabla 5). Si se cuenta con mayores recursos es posible pasar al sistema de tutorado vertical, aumentando los costos variables sobre el sistema anterior (horizontal), en $\$ 105.670$, permitiendo incrementar el ingreso neto, en \$268.080 adicionales, lo que representa una TRM adicional de $39 \%$. Los resultados anteriores sugieren que los tutorados en arvejas arbustivas se justifican en condiciones de alta precipitación durante el periodo de cultivo, porque generan mayores ingresos, como consecuencia de una mayor calidad de la producción. Este es el primer reporte de evaluación de tutorados en arveja arbustiva en Colombia; sin embargo, en arvejas volubles (Forero \& Ligarreto, 2009), buscando nuevas alternativas de tutorado, comparó el tutorado vertical frente al tutorado en arco, encontrando que, este último, no fue viable económicamente, por presentar menor rendimiento y mayores costos.

Tabla 4. Comparación de medias para el factor tutorado, en los sistemas TV: Tutorado vertical; TH: Tutorado horizontal; ST: Testigo sin tutor; en la variable de porcentaje de vainas sanas (\%VS).

\begin{tabular}{|c|c|}
\hline Sistema & \%VS \\
\hline TV & 78,2 A \\
\hline TH & 76,9 A \\
\hline ST & $56,46 \quad$ B \\
\hline
\end{tabular}

Promedios con letras distintas indican diferencia significativa, según la prueba de Tukey $(\mathrm{P} \leq 0,05)$.

Tabla 5. Ingreso total, ingreso neto, análisis de dominancia y tasa de retorno marginal (TRM), de los sistemas de tutorado en tres épocas de siembra.

\begin{tabular}{|c|c|c|c|c|c|c|c|c|c|c|c|c|}
\hline \multicolumn{2}{|c|}{ Tratamientos } & RTO & RTOC & \%San & $\begin{array}{l}\text { Ajuste } \\
\text { de RTO } \\
\text { x \%San }\end{array}$ & IT (\$) & IN (\$) & CV (\$) & $\begin{array}{l}\text { Domi- } \\
\text { nancia }\end{array}$ & $\begin{array}{l}\Delta \mathrm{IN} \\
(\mathbf{S})\end{array}$ & $\begin{array}{c}\Delta \mathrm{CV} \\
\mathbf{( \$ )}\end{array}$ & TRM \\
\hline \multirow{3}{*}{$\begin{array}{l}\text { Época } \\
\text { Id }\end{array}$} & ST & 5,5 & 6,04 & 58,98 & 3,56 & 8.220 .904 & 8.220 .904 & 0 & ND & - & & \\
\hline & $\mathrm{TH}$ & 6,1 & 6,04 & 78,84 & 4,76 & 10.984 .614 & 6.834 .735 & 4.154 .346 & D & - & - & - \\
\hline & TV & 6,5 & 6,04 & 80,23 & 4,84 & 11.169.229 & 6.760 .400 & 4.422 .426 & D & - & - & - \\
\hline \multirow{3}{*}{$\begin{array}{l}\text { Época } \\
\text { II }\end{array}$} & TV & 13,7 & 12,95 & 79,94 & 10,19 & 23.528 .307 & 19.373 .960 & 4.422 .426 & ND & 268.080 & 105.670 & $39 \%$ \\
\hline & $\mathrm{TH}$ & 13,3 & 12,95 & 78,69 & 10,35 & 23.902 .057 & 19.479 .630 & 4.154 .346 & ND & 4.154 .346 & 3.036 .603 & $73 \%$ \\
\hline & ST & 11,8 & 12,95 & 54,64 & 7,08 & 16.337 .358 & 16.337 .358 & 0 & ND & & - & - \\
\hline \multirow{3}{*}{$\begin{array}{l}\text { Época } \\
\text { III }\end{array}$} & ST & 9,9 & 10,21 & 73,18 & 7,48 & 17.242 .332 & 17.242 .332 & 0 & ND & - & - & - \\
\hline & TV & 11,4 & 10,21 & 74,42 & 7,6 & 17.534 .495 & 13.112 .068 & 4.422 .426 & D & - & - & - \\
\hline & TH & 9,3 & 10,21 & 55,76 & 5,7 & 13.137 .912 & 8.983 .566 & 4.154 .346 & D & - & - & - \\
\hline
\end{tabular}

RTO: Rendimiento (t.ha-1); RTOC: Rendimiento corregido (t.ha-1); \%San: Porcentaje de sanidad; IT: Ingreso total; IN: ingreso neto; CV: Costos variables; $\Delta \mathrm{CV}$ : Incremento en costos variables; $\Delta \mathrm{IN}$ : incremento en el ingreso neto; TRM: Tasa de retorno marginal; D: Dominado; ND: No dominado; TV: Tutorado vertical; TH: Tutorado horizontal; ST: Testigo sin tuto; \$: pesos colombianos. 
En conclusión, la línea UN6651 obtuvo los mejores resultados de rendimiento, sanidad, peso de vaina con grano y número de granos por vaina, en las épocas de siembra II (abril) y III (mayo), donde se presentó la mayor disponibilidad de agua para el cultivo. Igualmente, la línea UN5174 mostró mayor precocidad y alto rendimiento, siendo consistente, a través de las tres épocas de siembra. En los sistemas de tutorado horizontal y vertical, se presentaron los mayores promedios de sanidad de vaina. Por otra parte, las épocas de siembra II y III, con condiciones ambientales adecuadas, permitieron la mejor respuesta de rendimiento en las líneas estudiadas. Finalmente, la mayor viabilidad económica la mostró el sistema sin tutorado en las épocas I (marzo a junio) y III (mayo a agosto), de menor precipitación; sin embargo, la tasa de retorno marginal de los tutorados vertical y horizontal fue favorable para el agricultor en la época II (abril a julio), con lluvias más frecuentes.

Agradecimientos: Los autores agradecen al Grupo de Cultivos Andinos de la Facultad de Ciencias Agrícolas de la Universidad de Nariño, por su apoyo en el establecimiento de los ensayos de campo; al Centro Internacional de Producción Limpia, Sena Lope Seccional Nariño, por permitir el desarrollo de esta investigación en sus instalaciones y al I.A. M.Sc. Marino Rodríguez, por su asesoría en el diseño experimental y análisis estadístico. Conflicto de intereses: El manuscrito fue ejecutado, preparado y revisado con la participación de todos los autores, quienes declaramos que no existe conflicto de intereses que ponga en riesgo la validez de los resultados presentados. Financiación: Esta investigación fue financiada por la Vicerrectoría de Investigaciones, Posgrados y Relaciones Internacionales VIPRI, de la Universidad de Nariño, en el 2015.

\section{BIBLIOGRAFÍA}

1. BÉNÉZIT, M.; BIARNÈS, V.; JEUFFROY, M.; 2017. Impact of climate and diseases on pea yields: what perspectives with climate change? Oilseeds \& Fats Crops and Lipids. 24(1):1-9.

2. BUITRAGO, E.; DUARTE, C.; SARMIENTO, A. 2006. El cultivo de la arveja en Colombia. Federación Nacional de Cultivadores de Cereales y LeguminosasFENALCE y Fondo Nacional Cerealista. Produmedios. Bogotá. Colombia. 83p.

3. CASANOVA, E.; SOLARTE, J.; CHECA, O. 2012. Evaluación de cuatro densidades de siembra en siete líneas promisorias de arveja arbustiva (Pisum sativum L.). Rev. Ciencias Agrícolas. 29(2):129-140.

4. CELIS, A.; PRETT, G. 1995. Producción estival de arvejas en la costa interior en la Décima Región. Instituto de
Investigaciones Agropecuarias. Boletín Técnico Remehue. No. 232. Chile. 12p.

5. CHECA, O.; RODRÍGUEZ, M. 2015. Resistencia a oídio Erysiphe polygoni y rendimiento en arveja afila $P i$ sum sativum L. Rev. Temas Agrarios. 20(2):58-71.

6. DANE. 2016. Insumos y factores asociados a la producción agropecuaria: El cultivo de arveja en Colombia. Boletín mensual No.53. Disponible desde Internet en: www.dane.gov.co/files/investigaciones/agropecuario/sipsa/Bol_Insumos_nov_2016.pdf (con acceso $10 / 06 / 2017)$.

7. EGLI, D.B. 2017. Seed biology and yield of grain crops. CABI. University of Kentuchy. USA. 78p. DOI. 10.1079/9781780647708.0000.

8. ESMAIL, S.; ABDULKHALEQ, D.; HAMA, T.; KAREM, O. 2015. Correlation and path coefficient analysis in seven field pea (Pisum sativum L.) genotypes created by half diallel analysis in sulaimani region for $\mathrm{f} 2$ generation. Intern. J. Plant, Animal and Environmental Sc. 5(4):93-97.

9. FEDERACIÓN NACIONAL DE CULTIVADORES DE CEREALES Y LEGUMINOSAS -FENALCE-. 2006. El cultivo de la arveja en Colombia. Primera edición. Produmedios: Bogotá, D. C. 83 p.

10. FORERO, A.; LIGARRETO, G. 2009. Evaluación de dos sistemas de tutorado para el cultivo de la arveja voluble (Pisum sativum L.) en condiciones de la Sabana de Bogotá. Sociedad Colombiana De Ciencias Hortícolas-Scch, 81. DOI: https://doi.org/10.17584/ rcch.2009v3i1.1201.

11. GEORGIEVA, N.; NIKOLOVA, I.; KOSEV, V. 2015. Adaptation and stability of field peas (Pisum sativum L.) cultivars. J. Global Agriculture and Ecology. 3(1):1-10.

12. GOA, Y.; ASHAMO, M. 2014. Evaluation of field pea (Pisum sativum L.) genotypes performance for yield and yield components at five growing environments of southern Ethiopia. Current Res. Agric. Sc. 1(3):6576.

13. GONZÁLEZ, F.; LIGARRETO, G. 2006. Rendimiento de ocho genotipos promisorios de arveja arbustiva ( $P i-$ sum sativum L.) bajo sistema de agricultura protegida. Fitotecnia Col. 6(2):52-61.

14. HALLAUER, A.; CARENA, M.; MIRANDA, J. 2010. Quantitative genetics in maize breeding. Ed. Springer Sc. 
\& Bus. Media. New York. 664p. DOI. 10.1007/9781-4419-0766-0.

15. HERNÁNDEZ, M. 2002. Análisis económico de experimentos agrícolas con presupuestos parciales: Reenseñando el uso de este enfoque. Portal de revistas Nicaragua: La Calera. 2(2):45-47.

16. INSTITUTO DE HIDROLOGÍA, METEOROLOGÍA Y ESTUDIOS AMBIENTALES DE COLOMBIA -IDEAM-. 2016. Disponible desde Internet en: http://www. ideam.gov.co (con acceso 08/10/2016).

17. KHAN, T.; RAMZAN, A.; JILLANI, G.; MEHMOOD, T. 2013. Morphological performance of peas (Pisum sativum) genotypes under rainfed conditions of Potowar region. J. Agric. Res. 51(1):51- 60.

18. KUMAR, N.; ROOPA, G. 2014. Correlation coefficient analysis in field pea (Pisum sativum L.). Intern. J. Agric. Sc. and Res. (IJASR). 4:211-214.

19. LIGARRETO, G.; OSPINA, A. 2009. Análisis de parámetros heredables asociados al rendimiento y precocidad en arveja voluble (Pisum sativum L.) tipo Santa Isabel. Agron. Colomb. Vol. 27, Núm. 24p.

20. MERA, M.; KEHR, E.; MEJÍAS, J.; IHL, M.; BIFANI, V. 2007. Investigación arvejas (Pisum sativum L.) de vaina comestible "sugar snap": antecedentes y comportamiento en el sur de chile. Agric. Téc. 67(4):343352.

21. MISHRA, N. 2014. Growth and yield response of pea (Pisum.sativum $\mathrm{L}$.) to integrated nutrient management a review. J. Plant and Pest Sc. (2):87-95.
22. MOOT, D.J.; MCNEIL, D.L. 1995. Yield components, harvest index and plant type in relation to yield differences in field pea genotypes of partial resistance to field epidemics of Ascochyta blight of pea. Euphytica. 86:31-40.

23. MORENO, F. 2009. Respuesta de las plantas al estrés por déficit hídrico. Agronomía Colombiana. 27(2):179191.

24. PACHECO, CH.; VERGARA, H.; LIGARRETO, M. 2009. Clasificación de 85 accesiones de arveja (Pisum sativum L.), de acuerdo con su comportamiento agronómico y caracteres morfológicos. Agronomía Colombiana. 37(3):323-332.

25. PANTOJA, D.; MUÑOZ, K.; CHECA, O. 2014. Evaluación y correlación por componentes de rendimiento en líneas avanzadas de arveja Pisum sativum con gen afila. Rev. Agrícolas. 31(2):24-39.

26. RASAEI, A.; GHOBADI, M.E.; GHOBADI, M.; ABDYNIYA, K. 2011. The study of traits correlation and path analysis of the grain yield of the peas in semidry conditions in Kermanshah. Inter. Conf. on Food Engineering and Biotech. 9:246-249.

27. VALENCIA, A.; TIMANÁ, Y.; CHECA, O. 2012. Evaluación de 20 líneas de arveja (Pisum sativum L.) y su reacción al complejo de ascochyta. Rev. Ciencias Agrícolas. Universidad de Nariño. San Juan Pasto. 29(2):39-52.

Recibido: Marzo 28 de 2017

Aceptado: Agosto 30 de 2017

Cómo citar:

Checa Coral, O.E.; Bastidas Acosta, J.E.; Narváez Taimal, O.C. 2017. Evaluación agronómica y económica de arveja arbustiva (Pisum sativum L.) en diferentes épocas de siembra y sistemas de tutorado. Rev. U.D.C.A Act. \& Div. Cient. 20(2): $279-288$. 